\title{
Preferential reciprocal transfer of paternal/maternal DLK1 alleles to obese children: first evidence of polar overdominance in humans
}

\author{
Anne-Kathrin Wermter ${ }^{1,10}$, André Scherag ${ }^{2,3,10}$, David Meyre ${ }^{4}$, Kathrin Reichwald ${ }^{5,6}$, \\ Emmanuelle Durand $^{4}$, Thuy Trang Nguyen ${ }^{3}$, Kerstin Koberwitz ${ }^{7}$, Peter Lichtner ${ }^{7}$, \\ Thomas Meitinger ${ }^{7,8}$, Helmut Schäfer ${ }^{3}$, Anke Hinney ${ }^{5}$, Philippe Froguel ${ }^{4,9}$, \\ Johannes Hebebrand ${ }^{5}$ and Günter Brönner*,5
}

\begin{abstract}
${ }^{1}$ Department of Child and Adolescent Psychiatry, University of Marburg, Marburg, Germany; ${ }^{2}$ Institute of Medical Informatics, Biometry and Epidemiology, University of Duisburg-Essen, Essen, Germany; ${ }^{3}$ Institute of Medical Biometry and Epidemiology, University of Marburg, Marburg, Germany; ${ }^{4}$ Centre National de la Recherche Scientifique - Unité Mixte de Recherche 8090, Institute of Biology, Pasteur Institute, Lille, France; ${ }^{5}$ Department of Child and Adolescent Psychiatry, University of Duisburg-Essen, Essen, Germany; ${ }^{6}$ Leibniz Institute for Age Research - Fritz Lipmann Institute, Jena, Germany; ${ }^{7}$ Department of Human Genetics, Helmholtz Center Munich, Neuherberg, Germany; ${ }^{8}$ Department of Human Genetics, Technical University Munich, Munich, Germany; ${ }^{9}$ Section of Genomic Medicine and Genome Centre, Hammersmith Campus, Imperial College London, London, UK
\end{abstract}

DLK1 is part of the Notch signalling pathway that controls various developmental processes. A functional role for DLK1 in adipogenesis is suggested by several animal models. Interestingly, the DLK1 gene is imprinted in eutherian mammals. To study whether variations in DLK1 affect body weight in humans, we analysed 32 polymorphisms in a $109 \mathrm{~kb}$ genomic region encompassing DLK1 on human chromosome 14. In a study sample of 1025 French and German trio families comprised of both parents and extremely obese offspring we found a single nucleotide polymorphism (rs1802710) associated with child and adolescent obesity. Analysis of the allelic transmission pattern indicated the existence of polar overdominance, an unusual mode of non-mendelian inheritance in humans previously known from the callipyge mutation in sheep.

European Journal of Human Genetics (2008) 16, 1126-1134; doi:10.1038/ejhg.2008.64; published online 9 April 2008

Keywords: genetics; imprinting; obesity

\section{Introduction}

The delta-like 1 homologue (Drosophila) gene (DLK1), also known as PREF1, FA1 or $p G 2$, encodes a transmembrane protein containing epidermal growth factor-like repeats

*Correspondence: Dr G Brönner, Biozentrum, Universität Würzburg, Am Hubland, Würzburg 97074, Germany.

Tel: + 49931 8884440; Fax: + 49931 8887121;

E-mail: broenner@biozentrum.uni-wuerzburg.de

${ }^{10}$ These authors contributed equally to this work.

Received 6 September 2007; revised 5 February 2008; accepted 5 March 2008; published online 9 April 2008 homologous to the Notch/Delta/Serrate family of genes, which are involved in cell fate determination. Delta-like genes are conserved throughout the animal kingdom. Imprinting of DLK1 was probably acquired in eutherian mammals, ${ }^{1}$ which express only the paternal allele. ${ }^{2}$ While $D L K 1$ is widely expressed in embryogenesis, expression is turned off after birth in most tissues. DLK1 activity is important for many developmental processes including adipocyte differentiation. For example, it is highly expressed in 3T3-L1 preadipocytes but not detectable in differentiated adipocytes. ${ }^{3}$ Accordingly, adipogenesis is 
inhibited by constitutive expression of Dlk1 in 3T3-L1 cells, ${ }^{4}$ and increased by antisense RNA-mediated downregulation of $D l k 1.5$

These in vitro data are supported by rodent models. Mice expressing a $D l k 1$ transgene in adipose tissue are lean and show a decreased fat pad weight. ${ }^{6}$ Conversely, Dlk1 knockout mice are obese in addition to displaying other phenotypes such as growth retardation and skeletal malformations. ${ }^{7}$ The same phenotypes are observed in heterozygous animals if the knockout allele is inherited from the father, reflecting silencing of the maternal copy by imprinting. Heterozygotes having inherited the maternal null allele were normal.

Another interesting aspect of the DLK1 phenotypic spectrum has been discovered in sheep. Lambs, which express the callipyge phenotype, show a decreased fat mass and muscle fibre hypertrophy. ${ }^{8}$ The phenotype is caused by an apparent single locus mutation located distal to $D l k 1,{ }^{9}$ which is characterized by an unusual, non-mendelian mode of inheritance termed 'polar overdominance'.${ }^{10}$ Only heterozygous animals, which have inherited the callipyge mutation (CLPM) from their father $\left(C L P G^{\text {pat }} /+^{\text {mat }}\right.$ genotype) show 'visible' muscle hypertrophy when compared to the other three genotypes $\left(+{ }^{p a t} /+{ }^{m a t},+{ }^{p a t} / C L P G^{m a t}\right.$, and $\left.C L P G^{\text {pat }} / C L P G^{\text {mat }}\right)$. While in animals carrying the latter three genotypes, Dlk1 protein is not detectable in muscle tissue of 8-week-old lambs, the callipyge animals (CLPG ${ }^{\text {pat }}$ / $\left.+{ }^{\text {mat }}\right)$ do express Dlk1 in muscle suggesting an association between Dlk1 protein expression and the callipyge phenotype. ${ }^{11}$ A causative role of $D l k 1$ expression with respect to this phenotype has been shown by ectopic expression of Dlk1 in mice. That is, transgenic mice expressing Dlk1 under the control of the myosin promoter in skeletal muscle exhibit muscular hypertrophy resembling the callipyge phenotype of sheep. ${ }^{11}$

Evidence of polar overdominance has also been reported in pigs. ${ }^{12}$ Analysis of the parental origin of a silent polymorphism in Dlk1 revealed that paternal inheritance of one allele and maternal inheritance of the other allele in F2 offspring of two intercrossed lines is associated with decreased fatness and increased lean body mass.

Based on this evidence, we decided to analyse DLK1 with respect to a potential role in human body weight regulation. More specifically, we were interested in whether there would be parent-of-origin effects and, in particular, whether a pattern of polar overdominance would be detectable in humans. Therefore, we genotyped polymorphisms at the human DLK1 locus in two independent study samples and investigated a potential association with obesity. In order to be able to detect parent-of-origin effects, we chose to study trio families (two parents and one obese child) and employed statistical methods adapted to both detect imprinting effects and polar overdominance.

\section{Materials and methods Study subjects}

The family-based association and parent-of-origin studies in German samples were based on trios comprising unrelated children and adolescents with extreme obesity and both biological parents. The ascertainment strategy for these trio samples has been described previously. ${ }^{13}$ French trios were all Caucasians recruited in the Pediatric Unit of Jeanne de Flandre Hospital in Lille or through a national media campaign. ${ }^{14}$ The initial sample, comprising 359 obese children and adolescents (207 females; (mean \pm SD) body mass index (BMI) $31.77 \pm 6.07 \mathrm{~kg} / \mathrm{m}^{2}$; age $13.72 \pm 3.10$ years) and both biological parents, was genotyped for initially 3 and subsequently 29 polymorphisms.

The independent confirmatory sample comprised German and French trios. For both groups, the same phenotype definition was applied (BMI $\geq 99$ th percentile according to www.mybmi.de) allow for a better comparability of the samples. The German families consisted of 288 trios with obese children and adolescents (155 females; BMI, $33.93 \pm 5.39 \mathrm{~kg} / \mathrm{m}^{2}$; age $13.12 \pm 2.62$ years). The French sample comprised 378 trios with obese offspring (219 females; BMI, $31.99 \pm 6.32 \mathrm{~kg} / \mathrm{m}^{2}$; age $11.32 \pm 3.37$ years). In total, the initial and the independent confirmatory sample comprised 1025 obese children and adolescents (581 females; BMI, $32.46 \pm 6.05 \mathrm{~kg} / \mathrm{m}^{2}$; age $12.67 \pm 3.25$ years) and both biological parents. Screening for sequence variations was performed in a subgroup of the initial sample comprising 180 obese children and adolescents (101 females; BMI, $33.77 \pm 6.41 \mathrm{~kg} / \mathrm{m}^{2}$; age $14.38 \pm 2.82$ years). Written informed consent was given by all participants and, in the case of minors, by their parents. The ethics committee of the Universities of Marburg and Duisburg-Essen approved genotyping of obese subjects and their parents. The genetic study in French families was approved by ethical committee of the Centre Hospitalier Régional Universitaire in Lille.

\section{Genotyping of polymorphic variants}

In the initial sample three polymorphic variants were genotyped by PCR, following diagnostic restriction fragment length polymorphism (PCR-RFLP; Table 1). In total, 29 polymorphic variants were genotyped by PCR followed by a MassEXTEND reaction and subsequent matrix-assisted laser desorption/ionization time of flight mass spectrometry (MALDI-TOF MS) analysis according to the manufacturer's protocol (hME; Sequenom, San Diego, CA, USA; Table 1). The German trios in the confirmatory sample were genotyped by MALDI-TOF MS. In addition to MALDITOF MS genotyping, the polymorphic variant rs1802710 was genotyped by tetra-primer amplification refractory mutation system ${ }^{16}$ in a subgroup of the confirmatory sample. The French trios in the confirmatory sample were genotyped using Taqman assays (Applied Biosystems). Double genotyping was performed in 250 subjects for the 
Table 1 Genotyped polymorphic variants in a region encompassing $109 \mathrm{~kb}$ including $25-76 \mathrm{~kb} 3^{\prime}$ of the human $D L K 1 \mathrm{gene}$

\begin{tabular}{|c|c|c|c|c|c|c|c|}
\hline Nos. & $\begin{array}{l}\text { ID of } \\
\text { polymorphic } \\
\text { variants }^{\mathrm{a}}\end{array}$ & Position $^{\mathrm{b}}$ & Gene & Alleles $^{c}$ & $\begin{array}{l}\text { Intermarker } \\
\text { distance } \\
(b p)\end{array}$ & $\begin{array}{c}\text { Distance } \\
\text { DLK1 (bp) }\end{array}$ & $\begin{array}{c}\text { Observed } \\
\text { heterozygosities } \\
\text { (minor allele frequency) }^{\mathrm{d}}\end{array}$ \\
\hline 1 & rs17099615 & 100238191 & & $\mathrm{~T}$ & 3746 & 24848 & 0 \\
\hline 2 & rs7140792 & 100241936 & & $C>T$ & 874 & 21103 & $0.19(0.11)$ \\
\hline 3 & rs9635184 & 100242809 & & $\mathrm{C}>\mathrm{T}$ & 2743 & 20230 & $0.25(0.15)$ \\
\hline 4 & rs10151519 & 100245551 & put exon $1 D L K 1$ & $G>A$ & 170 & 17488 & $0.34(0.20)$ \\
\hline 5 & rs10873520 & 100245720 & put exon $1 D L K 1$ & $A>G$ & 803 & 17319 & $0.33(0.20)$ \\
\hline 6 & rs1555405 & 100246522 & put exon $1 D L K 1$ & $G>A$ & 1467 & 16517 & $0.39(0.25)$ \\
\hline 7 & rs 1004573 & 100247988 & & $C>G$ & 3371 & 15051 & $0.26(0.15)$ \\
\hline 8 & rs7155649 & 100251358 & & $C>A$ & 7127 & 11681 & $0.23(0.13)$ \\
\hline 9 & rs7155375 & 100258484 & & $\mathrm{C}>\mathrm{T}$ & 3569 & 4555 & $0.46(0.40)$ \\
\hline 10 & rs3759556 & 100262052 & & A & 542 & 987 & 0 \\
\hline 11 & rs11622172 & 100262593 & put exon $1 D L K 1$ & C & 551 & 446 & 0 \\
\hline 12 & rs10139403 & 100264314 & $D L K 1$ intron 1 & $G>A$ & 384 & & $0.42(0.34)$ \\
\hline 13 & rs6575799 & 100268170 & $D L K 1$ exon 4 & G & 10 & & 0 \\
\hline 14 & rs2273607 & 100268179 & $D L K 1$ exon 4 & G & 14 & & 0 \\
\hline 15 & rs1058006 & 100268192 & $D L K 1$ exon 4 & A & 303 & & 0 \\
\hline 16 & rs2273608 & 100268494 & $D L K 1$ intron 4 & $C>T$ & 1905 & & $0.16(0.08)$ \\
\hline 17 & rs 1802710 & 100270398 & $D L K 1$ exon 5 & $\mathrm{C}>\mathrm{T}$ & 216 & & $0.48(0.46)$ \\
\hline 18 & rs1058009 & 100270613 & $D L K 1$ exon 5 & $\mathrm{G}>\mathrm{A}$ & 3344 & & $0.11(0.06)$ \\
\hline 19 & rs876374 & 100273956 & DAT & $C>A$ & 4085 & 2742 & $0.48(0.44)$ \\
\hline 20 & rs1956742 & 100278040 & & $\mathrm{~T}>\mathrm{A}$ & 2871 & 6826 & $0.43(0.33)$ \\
\hline 21 & rs927257 & 100280910 & & $A>G$ & 3837 & 9696 & $0.44(0.33)$ \\
\hline 22 & rs2104070 & 100284746 & & $A>C$ & 5959 & 13532 & $0.24(0.15)$ \\
\hline 23 & rs11160604 & 100290704 & & $\mathrm{~T}>\mathrm{C}$ & 494 & 19490 & $0.24(0.15)$ \\
\hline 24 & rs2400938 & 100291197 & & $G>A$ & 3479 & 19983 & $0.12(0.06)$ \\
\hline 25 & rs2400940 & 100294675 & & $A>C$ & 8325 & 23461 & $0.30(0.19)$ \\
\hline 26 & rs1190820 & 100302999 & & $A>G$ & 6156 & 31785 & $0.50(0.48)$ \\
\hline 27 & rs941571 & 100309154 & & $C>T$ & 18355 & 37940 & $0.49(0.46)$ \\
\hline 28 & rs6575803 & 100327508 & $197 \mathrm{bp} 5^{\prime}$ of CLPM & $\mathrm{C}>\mathrm{T}$ & 2861 & 56294 & $0.18(0.10)$ \\
\hline 29 & rs8004581 & 100330368 & $2665 \mathrm{bp} 3^{\prime}$ of CLPM & $\mathrm{C}>\mathrm{T}$ & 4552 & 59154 & $0.27(0.16)$ \\
\hline 30 & rs4906013 & 100334919 & & $\mathrm{~T}>\mathrm{C}$ & 11638 & 63705 & $0.45(0.41)$ \\
\hline 31 & rs4906014 & 100346556 & IG-DMR & $A>C$ & 495 & 75342 & $0.31(0.20)$ \\
\hline 32 & rs1884539 & 100347539 & IG-DMR & $G>A$ & & 76325 & $0.46(0.38)$ \\
\hline
\end{tabular}

Abbreviations: CLPM, callipyge mutation in sheep; IG-DMR, intergenic differentially methylated region; Nos., numbers.

${ }^{a} \mathrm{dbSNP}$ (http://www.ncbi.nlm.nih.gov/SNP/).

${ }^{b}$ Human reference genome at the University of California Santa Cruz (UCSC) genome browser (http://genome.ucsc.edu/cgi-bin/hgGateway) (hg17). ${ }^{\mathrm{c}}$ Major allele $>$ minor allele.

In parents of the initial sample.

Except for rs9635184 (with $P=0.05$ ) no evidence for deviations from Hardy-Weinberg equilibrium in the founder data was obtained by PEDSTATS $0.6 .3 .^{15}$ (all exact $P \gg 0.10$ ).

polymorphic variant rs1802710 and the concordance rate was $100 \%$. In addition, no recurrent mendelian inconsistencies were detected in both the German and the French pedigrees using the PEDCHECK (version 1.1) program. $^{17}$

\section{Screening for sequence variations}

For analysis of $223 \mathrm{bp}$ of the human genomic region, which is orthologous to the ovine callipyge region containing the CLPM, single-strand conformation polymorphism analysis (SSCP) was performed as described previously. ${ }^{18}$ All amplicons were electrophoresed on 15\% acrylamide gels (37.5:1; Q-BIOgene, Heidelberg, Germany). Gels were run at room temperature for $5 \mathrm{~h}$ at $500 \mathrm{~V}$ and additionally at $4{ }^{\circ} \mathrm{C}$ for $17.5 \mathrm{~h}$ at $400 \mathrm{~V}$. Gels were silver stained. PCR products of individuals showing aberrant SSCP patterns were re-sequenced by a service laboratory (Seq/Lab, Göttingen, Germany).

\section{Statistical analysis}

Single marker family-based association analyses were performed using UNPHASED (version 3.0.5) ${ }^{19}$ and its implementation of the classical transmission disequilibrium test (TDT). ${ }^{20}$ The parent-of-origin effects were investigated by the parent-of-origin likelihood ratio test $\left(\mathrm{PO}-\mathrm{LRT}^{21}\right)$. This test allows for a comparison of paternal and maternal transmission rates while statistically controlling for prenatal (presumably non-genetic) maternal transmission effects. The parameters in the model $\left(I_{M}, S\right)$ were obtained by log-linear regression as implemented in SAS (version 8.2; SAS Institute Inc., Cary, NC, USA.). Under the global null hypothesis all parameters are expected to be 1 and a $P$-value for the global test can be derived (Table 2, column 4). Evidence for a paternally expressed copy is obtained by demonstrating that $I_{\mathrm{M}}<1$ meaning that a paternally derived copy of the allele is associated with a greater increase in risk than a maternally derived copy. 
Table 2 Analysis of parent-of-offspring effects by the PO-LRT parent-offspring likelihood ratio test (Weinberg ${ }^{21}$ ) of genotyped polymorphic variants in 359 German obesity trio families

\begin{tabular}{|c|c|c|c|c|c|c|c|c|c|c|c|c|}
\hline \multirow[t]{2}{*}{ Nos. } & \multirow{2}{*}{$\begin{array}{l}\text { Polymorphic } \\
\text { Variants }^{\mathrm{a}}\end{array}$} & \multirow{2}{*}{$\begin{array}{c}\text { Reference } \\
\text { Allele }\end{array}$} & \multicolumn{2}{|c|}{$L R T$} & \multicolumn{4}{|c|}{$I_{M}$} & \multicolumn{4}{|c|}{$S$} \\
\hline & & & $\chi^{2}$ & P-value & Estimate & & $\mathrm{Cl}$ & $\mathrm{P}$ (exact) & Estimate & & $\mathrm{Cl}$ & $\mathrm{P}$ (exact) \\
\hline 2 & rs7140792 & C & 2.20 & 0.33 & 1.37 & 0.58 & 3.25 & 0.55 & 0.68 & 0.38 & 1.19 & 0.20 \\
\hline 3 & rs9635184 & C & 0.10 & 0.95 & 0.92 & 0.42 & 1.99 & 0.95 & 1.02 & 0.61 & 1.71 & 1.00 \\
\hline 4 & rs10151519 & A & 0.21 & 0.90 & 1.01 & 0.50 & 2.05 & 1.00 & 1.06 & 0.66 & 1.69 & 0.90 \\
\hline 5 & rs10873520 & A & 0.12 & 0.94 & 0.96 & 0.47 & 1.97 & 1.00 & 1.07 & 0.67 & 1.71 & 0.86 \\
\hline 6 & rs1555405 & A & 0.15 & 0.93 & 0.96 & 0.47 & 1.97 & 1.00 & 1.06 & 0.68 & 1.66 & 0.88 \\
\hline 7 & rs1004573 & C & 0.13 & 0.94 & 1.08 & 0.51 & 2.32 & 0.97 & 0.92 & 0.55 & 1.53 & 0.82 \\
\hline 8 & rs7155649 & A & 6.07 & 0.05 & 0.59 & 0.27 & 1.28 & 0.20 & 1.82 & 1.08 & 3.13 & 0.02 \\
\hline 9 & rs7155375 & C & 0.40 & 0.82 & 1.13 & 0.58 & 2.21 & 0.81 & 0.89 & 0.60 & 1.32 & 0.61 \\
\hline 12 & rs10139403 & A & 0.89 & 0.64 & 0.80 & 0.40 & 1.58 & 0.59 & 1.20 & 0.81 & 1.79 & 0.40 \\
\hline 16 & rs2273608 & C & 5.97 & 0.05 & 2.20 & 0.85 & 5.90 & 0.11 & 0.47 & 0.23 & 0.91 & 0.02 \\
\hline 17 & rs1802710 & C & 8.39 & 0.02 & 0.43 & 0.22 & 0.82 & 0.01 & 1.66 & 1.13 & 2.47 & 0.01 \\
\hline 18 & rs1058009 & C & 0.38 & 0.83 & 1.15 & 0.40 & 3.33 & 0.96 & 0.84 & 0.44 & 1.59 & 0.66 \\
\hline 19 & rs876374 & A & 5.76 & 0.06 & 2.07 & 1.08 & 4.01 & 0.03 & 0.67 & 0.45 & 0.99 & 0.04 \\
\hline 20 & rs1956742 & A & 1.47 & 0.48 & 1.16 & 0.58 & 2.30 & 0.78 & 1.05 & 0.70 & 1.60 & 0.87 \\
\hline 21 & rs927257 & A & 1.20 & 0.55 & 0.87 & 0.44 & 1.71 & 0.78 & 0.96 & 0.64 & 1.45 & 0.92 \\
\hline 22 & rs2104070 & A & 1.30 & 0.52 & 0.67 & 0.32 & 1.41 & 0.34 & 1.20 & 0.76 & 1.92 & 0.47 \\
\hline 23 & rs11160604 & C & 1.30 & 0.52 & 1.49 & 0.71 & 3.15 & 0.34 & 0.83 & 0.52 & 1.32 & 0.47 \\
\hline 24 & rs2400938 & A & 2.44 & 0.29 & 2.03 & 0.74 & 5.71 & 0.20 & 0.64 & 0.31 & 1.28 & 0.23 \\
\hline 25 & rs2400940 & A & 3.76 & 0.15 & 1.81 & 0.91 & 3.61 & 0.09 & 0.69 & 0.44 & 1.06 & 0.09 \\
\hline 26 & rs1190820 & A & 4.08 & 0.13 & 1.27 & 0.66 & 2.47 & 0.54 & 0.74 & 0.50 & 1.08 & 0.12 \\
\hline 27 & rs941571 & C & 3.72 & 0.16 & 0.86 & 0.44 & 1.71 & 0.77 & 1.30 & 0.89 & 1.91 & 0.19 \\
\hline 28 & rs6575803 & C & 2.34 & 0.31 & 1.48 & 0.65 & 3.44 & 0.41 & 0.99 & 0.57 & 1.74 & 1.00 \\
\hline 29 & rs8004581 & C & 0.91 & 0.63 & 1.23 & 0.61 & 2.48 & 0.65 & 1.00 & 0.62 & 1.60 & 1.00 \\
\hline 30 & rs4906013 & C & 0.36 & 0.84 & 0.84 & 0.43 & 1.62 & 0.68 & 1.06 & 0.73 & 1.56 & 0.81 \\
\hline 31 & rs4906014 & A & 0.04 & 0.98 & 0.95 & 0.49 & 1.86 & 1.00 & 1.04 & 0.70 & 1.55 & 0.92 \\
\hline 32 & rs1884539 & A & 0.13 & 0.94 & 0.93 & 0.49 & 1.77 & 0.93 & 1.07 & 0.72 & 1.59 & 0.80 \\
\hline
\end{tabular}

Abbreviations: $I_{\mathrm{M}}$, parent-of-origin risk estimate ( $=1$ no effect, $<1$ paternal effect; $>1$ maternal effect); LRT, likelihood ratio test; Nos., numbers; $S$, relative risk estimates for the prenatal maternal effects assuming a co-dominant model.

adbSNP (http://www.ncbi.nlm.nih.gov/SNP/).

Monomorphic variants are not listed in this table.

Table 3 Modelling of genotype relative risks in all investigated obesity trios samples for rs 1802710 in exon 5 of DLK1. Likelihood ratio test $P$-values for each model, GRR point and interval estimates (95\% confidence interval) and the respective $P$-values for comparison to the reference category are presented

\begin{tabular}{|c|c|c|c|c|c|c|c|c|c|}
\hline \multirow[t]{2}{*}{ Model } & \multicolumn{3}{|c|}{$\begin{array}{c}\text { Initial } \\
\text { sample of } 359 \\
\text { German trios }\end{array}$} & \multicolumn{3}{|c|}{$\begin{array}{c}\text { Confirmatory } \\
\text { sample of } 666 \text { trios }\end{array}$} & \multicolumn{3}{|c|}{$\begin{array}{c}\text { Combined } \\
\text { sample of } 1025 \text { trios }\end{array}$} \\
\hline & $\begin{array}{c}L R T \\
(\mathrm{P} \text {-value })\end{array}$ & Estimate & $95 \% \mathrm{Cl}$ & $\begin{array}{c}\text { LRT } \\
(\mathrm{P} \text {-value })\end{array}$ & Estimate & $95 \% \mathrm{Cl}$ & $\begin{array}{c}L R T \\
\text { (P-value) }\end{array}$ & Estimate & $95 \% \mathrm{Cl}$ \\
\hline $\begin{array}{l}\text { No parent-of-origin effects } \\
\text { T/T } \\
\text { C/T } \\
\text { C/C }\end{array}$ & 0.93 & $\begin{array}{l}1 \\
0.96 \\
1.03\end{array}$ & $\begin{array}{l}- \\
(0.66-1.40) \\
(0.68-1.55)\end{array}$ & 0.34 & $\begin{array}{l}1 \\
1.21 \\
1.27\end{array}$ & $\begin{array}{c}- \\
(0.90-1.62) \\
(0.90-1.78)\end{array}$ & 0.73 & $\begin{array}{l}1 \\
1.08 \\
1.12\end{array}$ & $\begin{array}{l}- \\
(0.84-1.39) \\
(0.84-1.49)\end{array}$ \\
\hline $\begin{array}{l}\text { Imprinting effects } \\
\mathrm{T}^{\mathrm{mat}} / \mathrm{T}^{\text {pat }} \\
\mathrm{T}^{\mathrm{mat}} / \mathrm{C}^{\text {pat }} \\
\mathrm{C}^{\mathrm{mat}} / \mathrm{T}^{\text {pat }} \\
\mathrm{C}^{\mathrm{mat}} / \mathrm{C}^{\text {pat }}\end{array}$ & 0.07 & $\begin{array}{l}1 \\
1.47 \\
0.67 \\
1.05\end{array}$ & $\begin{array}{l}(0.89-2.43) \\
(0.42-1.07) \\
(0.69-1.59)\end{array}$ & 0.13 & $\begin{array}{l}1 \\
1.44 \\
0.93 \\
1.22\end{array}$ & $\begin{array}{l}(1.02-2.05) \\
(0.62-1.40) \\
(0.86-1.71)\end{array}$ & 0.03 & $\begin{array}{l}1 \\
1.39 \\
0.78 \\
1.08\end{array}$ & $\begin{array}{l}(1.03-1.89) \\
(0.55-1.09) \\
(0.81-1.45)\end{array}$ \\
\hline $\begin{array}{l}\text { Polar overdominance } \\
T^{\text {mat }} / T^{\text {pat }} \text { and } C^{\text {mat }} / C^{\text {pat }} \\
T^{\text {mat }} / C^{\text {pat }} \\
C^{\text {mat }} / T^{\text {pat }}\end{array}$ & 0.03 & $\begin{array}{l}1 \\
1.43 \\
0.65\end{array}$ & $\begin{array}{c}- \\
(0.93-2.20) \\
(0.43-0.98)\end{array}$ & 0.12 & $\begin{array}{l}1 \\
1.32 \\
0.82\end{array}$ & $\begin{array}{c}- \\
(0.97-1.80) \\
(0.58-1.16)\end{array}$ & 0.01 & $\begin{array}{l}1 \\
1.34 \\
0.74\end{array}$ & $\begin{array}{l}- \\
(1.02-1.75) \\
(0.55-0.99)\end{array}$ \\
\hline
\end{tabular}

Abbreviations: $\mathrm{Cl}$, confidence interval; LRT, likelihood ratio test.

A one-parameter model (with dummies coding 1 for $\mathrm{T}^{\mathrm{mat}} / \mathrm{C}^{\mathrm{pat}}$ and -1 for $\mathrm{C}^{\mathrm{mat}} / \mathrm{T}^{\mathrm{pat}}$ and 0 else) leads to an estimate of $1.35(95 \% \mathrm{Cl} 0.74-1.10)$ and a $P$-value of 0.003 in the combined sample.

${ }^{a}$ Reciprocal heterozygous genotypes express different phenotypes while homozygous genotypes express similar (normal) phenotypes. 
Values of $I_{M}>1$ indicate a maternally expressed copy. The estimate of the parameter $\mathrm{S}$ denotes the relative risks for maternally mediated genetic effects on the fetus assuming that the risk for a mother with one copy of the variant allele is midway between that for carriers of two or no copies. $^{22}$

In the presence of some evidence for parent-of-origin effects, we decided to investigate whether there is support for a polar overdominance mode of inheritance. This was done within the conditional logistic regression framework suggested by Cordell et al, ${ }^{23}$ which allows for an estimation of genotype relative risks (GRRs) conditional on parent-oforigin being inferable for the alleles at each locus. Note that GRR modelling differs from PO-LRT modelling due to differences in modelling; for an example, the parameter ' $\mathrm{S}$ ' will not be estimated and as a result the global likelihood ratio test $P$-values of the GRR models will not be identical to those obtained by PO-LRT. For coarse model comparisons, Akaike's information criterion (AIC) was used.

The analysis of between-marker linkage disequilibrium (LD) was done by use of Haploview version 3.2, ${ }^{24}$ applying the definition of 'haplotype blocks' as described by Gabriel et al. ${ }^{25}$ Parent-of-origin effects in haplotypes were investigated by FAMHAP version $16^{26}$ using the 'PAT' option, which allows to consider all combinations of tightly linked markers in nuclear families while incorporating corrections for multiple testing.

If not explicitly pointed out, all reported $P$-values are two sided and nominal. While exploration of the first sample lead to an obvious multiple testing problem, the second sample was only investigated for one single nucleotide polymorphism (SNP) by PO-LRT and the significance level for this test was $\alpha=0.05$ (two sided). In the initial sample, a transmission rate of 0.57 was observed in the informative fathers, that is in about $36 \%$ of all genotyped fathers. The power of the PO-LRT to confirm a parent-of-origin effect is mainly related to these parameters such that a sample size of 666 independent trios is estimated to yield a power of about $67 \%$ to detect a true transmission rate of 0.57 among an expected number of $0.36 \times 666=240$ informative fathers (one-sided binomial test with $\alpha=0.05$ using StatXact (version 5.0.3)).

\section{Results}

Study sample of 359 German obesity trio families To get an initial idea if DLK1 might be involved in body weight control in humans we initially genotyped three polymorphisms in 359 obesity trio families. One variant is located in the putative promoter region (rs3759556) and two in the translated region of exon five (rs1802710, rs1058009). rs3759556 was monomorphic in our study sample. When neglecting the parental origin of the transmitted alleles, the TDT revealed no evidence of linkage and allelic association with obesity for rs1802710 and rs1058009. However, for rs1802710, a coding synonymous variation, stratification based on parental origin showed a more frequent paternal transmission of the C-allele to obese children (75 transmitted versus 56 nontransmitted among heterozygous fathers). Consequently, we applied the PO-LRT, which is suitable for detection of parent-of-origin effects. ${ }^{21}$ For rs 1802710 , this test indicated evidence for an association with obesity $(P=0.015)$ with an increased paternal transmission of the C-allele to the affected offspring $\left(I_{M}=0.43 ; 95 \% \quad C I, 0.22-0.82\right.$, see 'Materials and methods' and Table 2 for details) and some evidence for maternally mediated genetic effects as well $(\mathrm{S}=1.66 ; 95 \% \mathrm{CI}, 1.13-2.47)$. No such pattern was found for rs1058009 (Table 2).

To detect signs of polar overdominance, we calculated the GRRs for rs1802710 taking the $\mathrm{T}^{\text {mat }} / \mathrm{T}^{\text {pat }}$ genotype as reference category (based on the PO-LRT results). Table 3 shows a summary of these calculations. The results for the heterozygous genotypes $\mathrm{T}^{\mathrm{mat}} / \mathrm{C}^{\mathrm{pat}}$ and $\mathrm{C}^{\mathrm{mat}} / \mathrm{T}^{\mathrm{pat}}$ are consistent with the imprinting hypothesis for $D L K 1$, that is only the paternally transmitted allele is expressed, and are an alternative illustration of the PO-LRT results reported above. Note however that the relative risk for carriers of the homozygous $\mathrm{C}^{\mathrm{mat}} / \mathrm{C}^{\text {pat }}$ genotype is not increased compared to the reference genotype. In the case of polar overdominance though, the increased risk would not be present for $C^{\text {mat }} / C^{\text {pat }}$ genotype carriers. Thus, the observed pattern of inheritance in our sample is congruent with the model of polar overdominance and reminiscent of the callipyge pattern originally observed in sheep. This result prompted us to further investigate the DLK1 genomic region including $5^{\prime}$ - and $3^{\prime}$-flanking regions.

In order to identify potentially causative mutations which are in LD with rs1802710, we analysed 29 additional SNPs in a $109 \mathrm{~kb}$ region encompassing DLK1 (Figure 1). In total, 10 of these SNPs were located in the $5^{\prime}$-region of DLK1, 5 SNPs in the transcribed region including introns and 14 SNPs in the $3^{\prime}$-region. The $3^{\prime}$-region also contains the sequences homologous to the ovine callipyge region ${ }^{27}$ and the murine intergenic differentially methylated region (IG-DMR). ${ }^{28}$ The observed heterozygosities and the minor allele frequencies of all analysed SNPs are listed in Table 1. Five of the presumed polymorphisms were found to be monomorphic in our study sample. For the remaining 24 SNPs, the calculation of PO-LRT did not reveal strong evidence for an association with obesity and parent-oforigin effects (rs7155649 $P=0.048$, all other $P>0.05$; Table 2).

\section{Screening of the callipyge homologous region}

In sheep, the CLPM is located $56 \mathrm{~kb} 3^{\prime}$ of $D l k 1$, and, interestingly, the adjacent sequence is highly conserved between human and sheep (Figure 2a). To find out whether a callipyge (CLPG)-like mutation occurs in our patient sample we analysed an $86 \mathrm{bp}$ genomic fragment homo- 

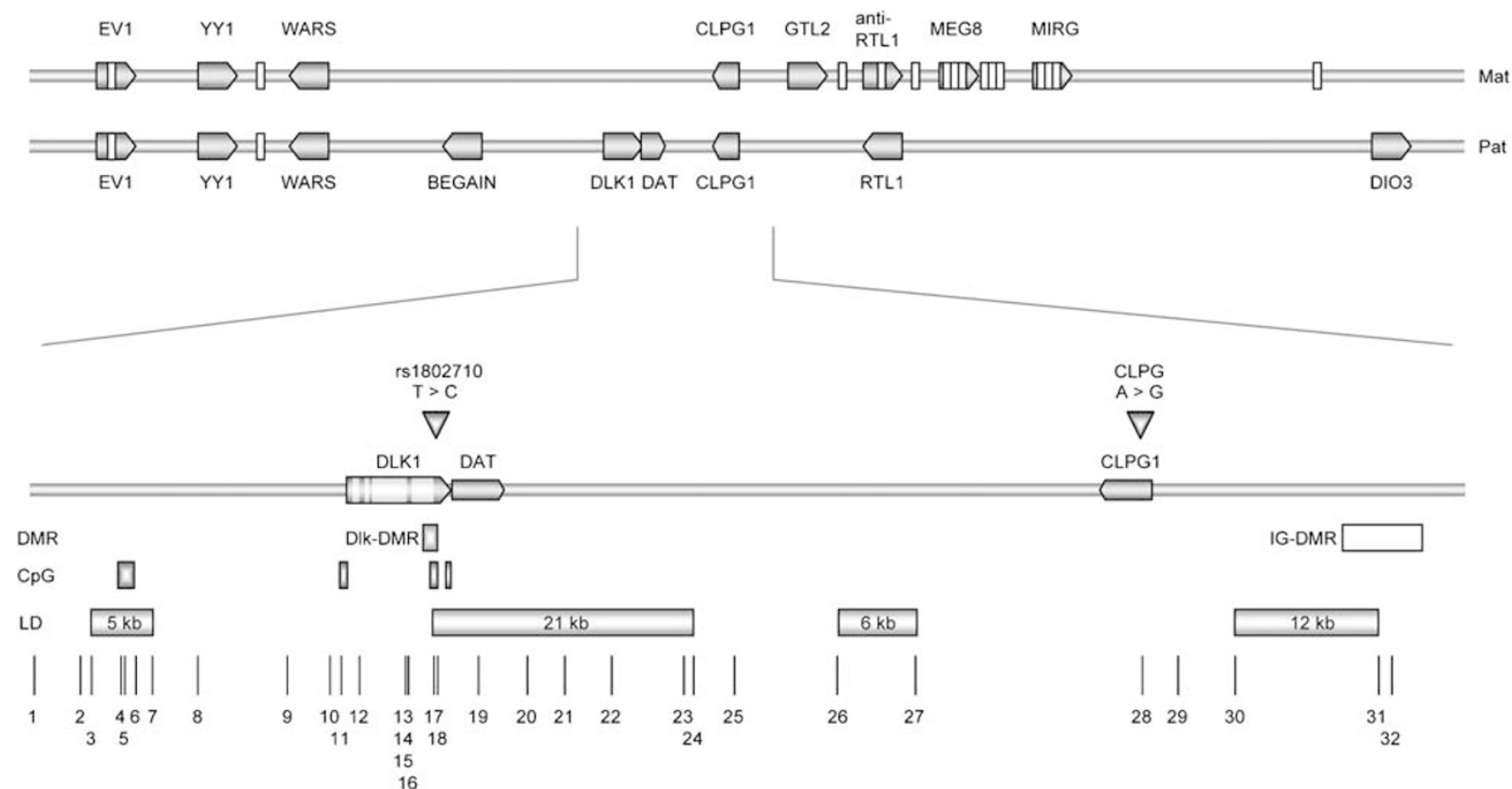

Figure 1 (Top) Schematic representation of the imprinted chromosomal region surrounding the human DLK1 gene. (Bottom) DLK1 region analysed in the current study. Arrows show transcription units (lighter areas within DLK1 depict introns). White boxes indicate single or clustered snoRNAs or miRNAs. Triangles point to the positions of the rs 1802710 polymorphism and of the callipyge (CLPG) mutation, respectively. Numbered vertical lines represent the polymorphisms as listed in Table 1. DMR, differentially methylated region; CpG, CpG island; LD, linkage disequilibrium block.

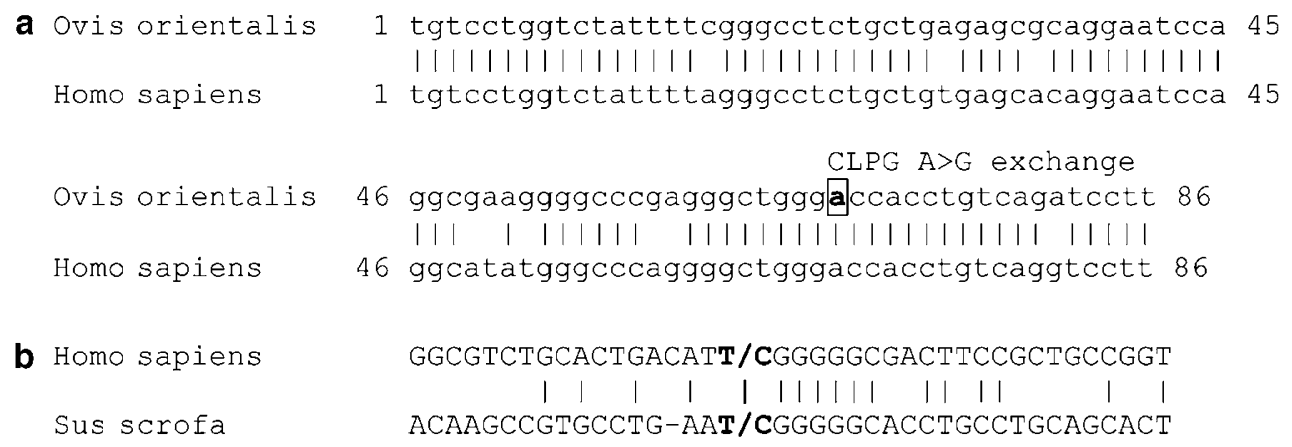

Figure 2 (a) Alignment of human and ovine 86-bp genomic sequences surrounding the callipyge (CLPG) mutation. (b) Sequence similarity of fragments encompassing the exon 5 polymorphism in pig $^{12}$ and rs1802710 in humans. The positions of the CLPG mutation and the exon 5 polymorphisms are indicated in bold.

logous to the ovine CLPG region by SSCP analysis. However, screening of 180 obese patients did not reveal any mutations.

\section{Linkage disequilibrium and haplotype analyses}

The haplotype structure of the $109 \mathrm{~kb}$ genomic region encompassing DLK1 was analysed in 359 obesity trios. Four relatively short 'LD blocks' were identified. rs1802710 was located at the edge of LD block 2 (Figure 1). None of the relevant haplotypes showed stronger evidence for an obesity association or parent-of-origin effect than rs1802710 alone. Furthermore, testing parent-of-origin effects in all combinations of possible haplotypes among SNPs within LD block 2 revealed that the top 4\% (sorting by nominal $P$-values) of all 255 combinations always included rs1802710.

\section{Confirmatory sample of German and French obesity trio families}

The results in our first study sample indicated a small effect size and suggested that a very large sample would be required to confirm the initial finding. In that light the paucity of available obesity trios was challenging especially, because case-control designs cannot detect the 
observed mode of inheritance. Therefore, in an effort to test the hypothesis of parent-of-origin effects and of polar overdominance, which was based on the animal data and our initial results for rs1802710, we gathered and combined two additional independent sample sets comprising 666 trios of European descent with extremely obese offspring from France and Germany (see 'Materials and methods' for a detailed description).

A similar transmission pattern as in the first sample was observed in the German and French trios. As found in the first study sample, the standard TDT indicated no association with obesity $(P=0.19)$. Again, stratification by parental origin revealed a more frequent paternal transmission of the C-allele (133 transmitted and 100 nontransmitted). The PO-LRT (LRT, $P=0.04 ; I_{\mathrm{M}}=0.62,95 \%$ CI, 0.40-0.97) again supported the initial observation of parent-of-origin effects while the previously observed maternally mediated genetic effects was now absent ( $\mathrm{S}=1.16,95 \% \mathrm{CI}, 0.90-1.51$ ). In addition, the GRRs (with $\mathrm{T}^{\text {mat }} / \mathrm{T}^{\text {pat }}$ as reference genotype) displayed a similar pattern as in the first sample (Table 3 ).

In order to obtain a more precise estimate of allele transmissions and the related obesity risks, we pooled the data of the first and confirmatory sample totalling in 1025 obesity trios, calculated the PO-LRT for rs1802710 and determined the GRR. The $P$-value of the global LRT was 0.002 for the PO-LRT, due to a preferential transmission of paternal C-alleles to obese offspring $\left(I_{M}=0.55,95 \% \mathrm{CI}\right.$, $0.38-0.78)$. The results of the GRR calculations are presented in Table 3 and indicated a relative risk for carriers of the $\mathrm{T}^{\text {mat }} / \mathrm{C}^{\text {pat }}$ genotype of 1.34 and for carriers of the $\mathrm{C}^{\text {mat }} / \mathrm{T}^{\text {pat }}$ genotype of 0.74 (global $P=0.006$ ). Our data can be best described (smallest AIC) by assuming a polar overdominance mode of inheritance.

\section{Discussion}

Several model systems support an association of the imprinted DLK1 gene with adiposity. Adipogenesis of 3T3-L1 cells is inhibited by Dlk1 expression ${ }^{4}$ and enhanced by antisense RNA-mediated downregulation of $D l k 1 .^{5}$ Knockout mice lacking both or only the paternally inherited Dlk1 gene are obese. CLPG sheep display muscle hypertrophy and reduced fatness. ${ }^{8}$ In pig, analysis of a silent polymorphism in exon 5 of Dlk1 suggested the existence of polar overdominance too. Animals having inherited one allele of this polymorphism from the father and the other allele from the mother were less fat and had increased lean body mass compared with all other genotypes. $^{12}$

In our study in humans, a synonymous polymorphism (rs1802710) in the fifth exon of DLK1, only $93 \mathrm{bp} \mathrm{5'}$ of the relevant polymorphism in the orthologous pig sequence, was associated with extreme obesity. This was revealed by a hypothesis-driven analysis assuming an inheritance pattern compatible with polar overdominance, which was originally reported in sheep. Initially, genotyping was done in a study sample of 359 obesity trios and multiple genetic markers and multiple genetic models were evaluated even though one may argue that based on the animal data there is a hierarchy among the tests performed (that is the test for polar overdominance should be the most appropriate one). To address the problem of overfitting, a second and independent sample of 666 obesity trios was genotyped for the relevant marker (rs1802710) to validate the initial finding. The combined sample $(n=1025)$ represents one of the largest obesity trio samples to be analysed until today. Analyses of the transmission pattern of rs1802710 alleles to the affected offspring in both independent trio samples revealed a significant parent-of-origin effect reflecting the known silencing of the maternally inherited copy by imprinting. ${ }^{2}$ Unexplainable by imprinting alone, we also observed a transmission pattern that is best explained on the basis of polar overdominance.

Due to the lack of body composition data we are unable to assess potential effects of SNP rs1802710 on fat mass and lean body mass separately. Based on the sheep and pig data, the polymorphism could even be associated with opposed values of fat and lean body mass that could obscure the phenotype when looking solely at BMI. Certainly, further confirmation in independent study samples would be desirable to reduce the risk of a false positive finding. The analysed 1025 German and French obesity trios, however, represented all trios with extremely obese offspring available to us. Because of the special mode of inheritance a replication cannot be done by case-control designs. Furthermore, family samples for a powerful replication need to be of substantial size as we estimated a rather small genetic effect size, which holds true for other common polymorphisms involved in body weight regulation. Assuming that our finding is not a false positive one, how then can our result be explained and integrated into existing theories?

Whether the $\mathrm{T}>\mathrm{C}$ exchange at rs1802710 has functional relevance is unclear. The exchange is silent and analysis of the fifth exon of DLK1 with the ESEfinder program (release 2.0) did not provide evidence for impaired or newly created splice sites by any of the analysed polymorphic variants. Theoretically, it is conceivable that rs1802710 is linked to a proximate mutation changing amino acids. However, this seems rather unlikely since it is difficult to imagine how changes at the amino-acid level could evoke a phenomenon such as polar overdominance characterized by phenotypically normal homozygous mutation carriers.

A look at callipyge sheep suggests alternatives to explain the observed rs1802710 results. The relationship between different models of gene action and the slaughter phenotypes of callipyge lambs ${ }^{8}$ suggested that the inheritance of CLPG is best explained by polar overdominance: 
Reciprocal heterozygous genotypes express different phenotypes (polar), and the two homozygous genotypes produce similar, normal phenotypes (overdominance). ${ }^{29}$ Although the exact molecular activities underlying the CLPG phenotype are still not understood, the identification of the CLPG mutation ${ }^{9}$ allowed to better analyse its molecular effects. The CLPG mutation is an A $>\mathrm{G}$ transition in the intergenic region between Dlk1 and Gtl2. The mutation changes the expression level of many of the genes in the imprinting region (Figure 1). However, the only known molecular marker that is perfectly correlated with the callipyge muscle phenotype is Dlk1 protein. ${ }^{11}$ Only in muscles of the $+{ }^{\text {mat }} / \mathrm{CLPG}^{\text {pat }}$ genotype Dlk1 protein is expressed whereas in muscles of all other genotypes (including $\mathrm{CLPG}^{\text {mat }} / \mathrm{CLPG}^{\text {pat}}$ ) no Dlk1 protein was detectable. These findings resulted in a polar overdominance model, in which CLPG $^{\text {pat }}$ expresses a muscle hypertrophy effector (Dlk1) and CLPG ${ }^{\text {mat }}$ expresses a repressor that inhibits translation of Dlk1. This way, only the + mat/ $\mathrm{CLPG}^{\text {pat }}$ genotype would produce Dlk1 protein. The repressor would likely be one of the non-coding RNAs transcribed from the maternal chromosome, possibly a microRNA, which would bind to DLK1 RNA and prevent its translation. ${ }^{11}$ Of course, alternative models cannot be excluded at this point.

Because of the callipyge example, we had screened the highly conserved human region corresponding to the region flanking the CLPG mutation in sheep for sequence variations that might be in LD with rs1802710. In 360 analysed human chromosomes, we could not detect any sequence variation. Furthermore, genotyping of SNPs located close to the CLPG orthologous position did not reveal any parent-of-offspring effects and association with obesity. This is in accordance with our observation that all other SNPs that we genotyped in the DLK1 region (Figure 1) and all analysed haplotypes did not show better obesity association than rs 1802710 alone. A similar situation exists in pigs. Here, a silent polymorphism was detected in the fifth exon of Dlk1, which is associated with decreased fat deposition and increased lean muscle mass. Again, sequencing of pig DNA fragments homologous to the Dlk1-Gtl2 region did not reveal additional polymorphisms. ${ }^{12}$ This suggests that in both pig and human the exon 5 polymorphisms are either in LD with a causative mutation most likely outside of the analysed region or that the altered DNA sequences are causative themselves.

An alignment of pig and human DLK1 sequences shows that both polymorphisms are located closely together, only $93 \mathrm{bp}$ apart. More interestingly, the sequences immediately adjacent to both SNPs are very similar (Figure $2 b$ ). Both polymorphisms are part of a TGGGGGC stretch and the $\mathrm{T}>\mathrm{C}$ exchange creates a new $\mathrm{CpG}$ dinucleotide. In human, this region is located inside of a CpG island (Figure 1). The sequence identity around the polymorphisms suggests that this site could be the target site of transcriptional or post- transcriptional regulator(s) as postulated in the aforementioned polar overdominance model. Obviously, it would be desirable to measure expression levels of $D L K 1$ in adipose tissue in vivo. However, in humans this task is not easily accomplished. A sufficient number of high quality tissue probes grouped into the four different genotypes would be necessary for this experiment. Acquisition of such adipocyte probes is not trivial, particularly if children are involved. Furthermore, the known biological roles of DLK1 suggest that it functions early during development, when access to suitable tissue probes is not readily possible.

Despite the many open questions, further analysis of the potential link between imprinting controlled gene regulation and obesity could improve our understanding of this complex phenotype. Mice generated by somatic cloning showed increased body fat and body size, possibly caused by imprinting defects induced during the cloning process. ${ }^{30}$ Analysis of gene expression in cloned mice revealed that $D l k 1$ is one of the genes with consistently and significantly reduced expression levels. ${ }^{31}$ Interestingly, even in vitro fertilized mice are heavier than their background stock mice. ${ }^{30}$ In view of the sporadic reports about increased incidence of imprinting defects (eg Angelman or Beckwith-Wiedemann syndrome) in children born after in vitro fertilization, ${ }^{32}$ it may be indicated also to monitor long-term development such as increased adiposity at later ages of in vitro fertilized humans.

In conclusion, we have shown that the human DLK1 gene is associated with extreme early-onset obesity. This association is only apparent when imprinting of the gene is considered. The allelic transmission pattern supports the existence of polar overdominance in humans. We hope that our finding encourages other groups to attempt a replication.

\section{Acknowledgements}

We thank Gudrun Höhn, Gerti Gerber and Jitka Andrä for expert technical assistance. This work was supported by grants from the European Union (FP6 LSHMCT-2003-503041), the Bundesministerium für Bildung und Forschung (NGFN2 01GS0482, 01GSO483, 01GR0460) and the Deutsche Forschungsgemeinschaft (HE 1446/4-1).

\section{References}

1 Weidman JR, Maloney KA, Jirtle RL: Comparative phylogenetic analysis reveals multiple non-imprinted isoforms of opossum Dlk1. Mamm Genome 2006; 17: 157-167.

2 Wylie AA, Murphy SK, Orton TC, Jirtle RL: Novel imprinted DLK1/GTL2 domain on human chromosome 14 contains motifs that mimic those implicated in IGF2/H19 regulation. Genome Res 2000; 10: $1711-1718$.

3 Smas CM, Kachinskas D, Liu CM, Xie X, Dircks LK, Sul HS: Transcriptional control of the pref-1 gene in 3T3-L1 adipocyte differentiation. Sequence requirement for differentiation-dependent suppression. J Biol Chem 1998; 273: 31751-31758. 
4 Smas CM, Sul HS: Pref-1, a protein containing EGF-like repeats, inhibits adipocyte differentiation. Cell 1993; 73: $725-734$.

5 Smas CM, Chen L, Zhao L, Latasa MJ, Sul HS: Transcriptional repression of pref-1 by glucocorticoids promotes 3T3-L1 adipocyte differentiation. J Biol Chem 1999; 274: 12632-12641.

6 Lee K, Villena JA, Moon YS et al: Inhibition of adipogenesis and development of glucose intolerance by soluble preadipocyte factor-1 (Pref-1). J Clin Invest 2003; 111: 453-461.

7 Moon YS, Smas CM, Lee K et al: Mice lacking paternally expressed Pref-1/Dlk1 display growth retardation and accelerated adiposity. Mol Cell Biol 2002; 22: 5585-5592.

8 Freking BA, Keele JW, Nielsen MK, Leymaster KA: Evaluation of the ovine callipyge locus: II. Genotypic effects on growth, slaughter, and carcass traits. J Anim Sci 1998; 76: 2549-2559.

9 Freking BA, Murphy SK, Wylie AA et al: Identification of the single base change causing the callipyge muscle hypertrophy phenotype, the only known example of polar overdominance in mammals. Genome Res 2002; 12: 1496-1506.

10 Cockett NE, Jackson SP, Shay TL et al: Polar overdominance at the ovine callipyge locus. Science 1996; 273: 236-238.

11 Davis E, Jensen $\mathrm{CH}$, Schroder HD et al: Ectopic expression of DLK1 protein in skeletal muscle of padumnal heterozygotes causes the callipyge phenotype. Curr Biol 2004; 14: $1858-1862$.

12 Kim KS, Kim JJ, Dekkers JC, Rothschild MF: Polar overdominant inheritance of a DLK1 polymorphism is associated with growth and fatness in pigs. Mamm Genome 2004; 15: 552-559.

13 Hebebrand J, Himmelmann GW, Heseker $H$, Schafer $H$, Remschmidt $\mathrm{H}$ : Use of percentiles for the body mass index in anorexia nervosa: diagnostic, epidemiological, and therapeutic considerations. Int J Eat Disord 1996; 19: 359-369.

14 Bouatia-Naji N, De Graeve F, Brönner G et al: INS VNTR is not associated with childhood obesity in 1,023 families: a familybased study. Obesity 2008, (In press).

15 Wigginton JE, Abecasis GR: PEDSTATS: descriptive statistics, graphics and quality assessment for gene mapping data. Bioinformatics 2005; 21: $3445-3447$.

16 Ye S, Dhillon S, Ke X, Collins AR, Day IN: An efficient procedure for genotyping single nucleotide polymorphisms. Nucleic Acids Res 2001; 29: E88.

17 O'Connell JR, Weeks DE: PedCheck: a program for identification of genotype incompatibilities in linkage analysis. Am J Hum Genet 1998; 63: 259-266.

18 Hinney A, Schmidt A, Nottebom K et al: Several mutations in the melanocortin-4 receptor gene including a nonsense and a frameshift mutation associated with dominantly inherited obesity in humans. J Clin Endocrinol Metab 1999; 84: 1483-1486.
19 Dudbridge F: Pedigree disequilibrium tests for multilocus haplotypes. Genet Epidemiol 2003; 25: 115-121.

20 Spielman RS, McGinnis RE, Ewens WJ: Transmission test for linkage disequilibrium: the insulin gene region and insulindependent diabetes mellitus (IDDM). Am J Hum Genet 1993; 52: 506-516.

21 Weinberg CR: Methods for detection of parent-of-origin effects in genetic studies of case-parents triads. Am J Hum Genet 1999; 65: $229-235$.

22 Infante-Rivard C, Weinberg CR: Parent-of-origin transmission of thrombophilic alleles to intrauterine growth-restricted newborns and transmission-ratio distortion in unaffected newborns. Am J Epidemiol 2005; 162: 891-897.

23 Cordell HJ, Barratt BJ, Clayton DG: Case/pseudocontrol analysis in genetic association studies: a unified framework for detection of genotype and haplotype associations, gene-gene and geneenvironment interactions, and parent-of-origin effects. Genet Epidemiol 2004; 26: 167-185.

24 Barrett JC, Fry B, Maller J, Daly MJ: Haploview: analysis and visualization of LD and haplotype maps. Bioinformatics $2005 ; 21$ : $263-265$.

25 Gabriel SB, Schaffner SF, Nguyen $\mathrm{H}$ et al: The structure of haplotype blocks in the human genome. Science 2002; 296: $2225-2229$.

26 Becker T, Knapp M: A powerful strategy to account for multiple testing in the context of haplotype analysis. Am J Hum Genet 2004; 75: $561-570$.

27 Charlier C, Segers K, Karim L et al: The callipyge mutation enhances the expression of coregulated imprinted genes in cis without affecting their imprinting status. Nat Genet 2001; 27: $367-369$.

28 Takada S, Paulsen M, Tevendale M et al: Epigenetic analysis of the Dlk1-Gtl2 imprinted domain on mouse chromosome 12: implications for imprinting control from comparison with Igf2H19. Hum Mol Genet 2002; 11: 77-86.

29 Freking BA, Keele JW, Beattie CW et al: Evaluation of the ovine callipyge locus: I. relative chromosomal position and gene action. J Anim Sci 1998; 76: 2062-2071.

30 Tamashiro KL, Wakayama T, Akutsu $\mathrm{H}$ et al: Cloned mice have an obese phenotype not transmitted to their offspring. Nat Med 2002; 8: 262-267.

31 Humpherys D, Eggan $\mathrm{K}$, Akutsu $\mathrm{H}$ et al: Abnormal gene expression in cloned mice derived from embryonic stem cell and cumulus cell nuclei. Proc Natl Acad Sci USA 2002; 99: $12889-12894$.

32 Devroey P, Van Steirteghem A: A review of ten years experience of ICSI. Hum Reprod Update 2004; 10: 19-28 